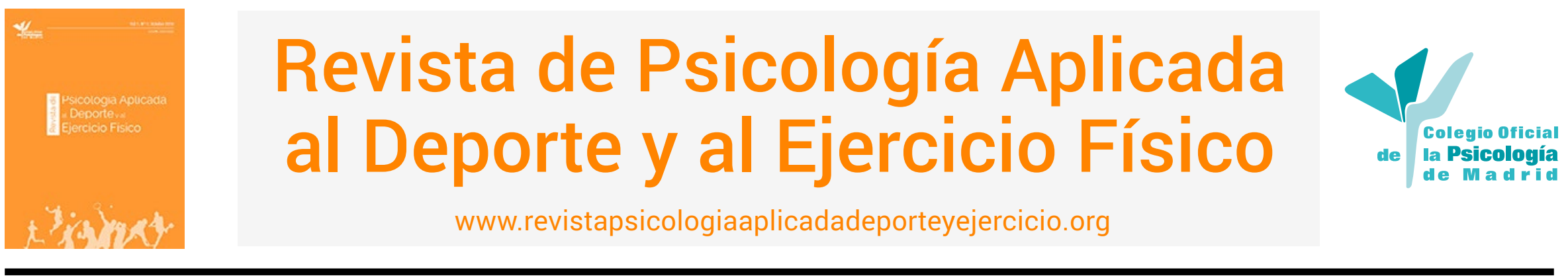

\title{
Aplicación del modelo de las 5Cs para la formación de entrenadores: Un ejemplo de práctica basada en la evidencia.
}

\author{
Marta Borrueco ${ }^{1,2}$, Yago Ramis ${ }^{1,2}$, Susana Pallarès ${ }^{1,2}$ y Jaume Cruz ${ }^{1,2}$ \\ 1 Grup d'Estudis en Psicologia de l'Esport i de l'Activitat Física (GEPE); Universitat Autònoma de Barcelona, España \\ 2 Institut de Recerca de l'Esport; Universitat Autònoma de Barcelona, España
}

RESUMEN: El presente trabajo tiene el objetivo de desarrollar e implementar un programa de formación basado en la evidencia dirigido a entrenadores de un club de hockey sobre patines, con el fin de optimizar el desarrollo psicosocial de sus deportistas. Esta experiencia profesional explica cómo a partir de una demanda del club, el Grup d'Estudis en Psicologia de l'Esport i de l'Activitat Física (GEPE) desarrolló un programa de formación adaptado a sus características y necesidades. Para desarrollar el programa, se siguieron las fases propuestas por el Modelo GEPE de PBE (i.e., Práctica Basada en la Evidencia): (a) actualización documental mediante la revisión de evidencia científica sobre la influencia del entrenador en los deportistas, (b) análisis de necesidades del club y de sus entrenadores, (c) desarrollo y aplicación de un programa de formación basado en el Modelo de las 5Cs (i.e., compromiso, comunicación, concentración, control, y confianza) para aumentar las habilidades de los entrenadores en la promoción del desarrollo psicosocial de sus deportistas, (d) evaluación del programa de formación, y (e) seguimiento de los efectos del programa a lo largo del tiempo. El programa de formación constó de 5 talleres teórico-prácticos, uno para cada C, en los que participaron entre 6 y 12 entrenadores por taller. La satisfacción de los participantes, evaluada tras cada taller, mostró que los talleres habían cubierto las expectativas de los entrenadores y que, además, consideraban que el taller brindaba un contexto ideal para compartir experiencias con sus colegas. Como áreas de mejora, consideraron que se debían añadir más contenidos prácticos. Este trabajo se plantea como un ejemplo de integración de la evidencia científica en la práctica aplicada para los profesionales de la psicología del deporte, dotando de rigor su práctica profesional.

PALABRAS CLAVES: desarrollo psicosocial, práctica basada en la evidencia, entrenador/a, programa de formación, desarrollo juvenil positivo.

\section{Applying the 5Cs model for coach training: An example of evidence-based practice}

ABSTRACT: The main objective of this work was to develop and implement an evidence-based education programme aimed at enhancing roller-hockey coaches' coaching effectiveness to optimize players' psychosocial development. This professional experience describes how the GEPE (Research Group in Sport and Exercise Psychology) developed their consultancy work after receiving the demand from the roller-hockey club by following the five steps proposed in the GEPE Model of Evidence-Based Practice: (a) literature update about scientific and applied evidence regarding the coaches' influence in their players, (b) assessing the needs of the club and the coaches, (c) development of an education program with the objective of teaching coaches how to promote the 5Cs (i.e., commitment, communication, concentration, control, and confidence) of the players' psychosocial development, (d) assessment of the program, and

Financiación: Este trabajo se ha realizado en parte gracias a la beca FI-DGR (2018FI_B_01099) de la Generalitat de Catalunya y al proyecto RTI2018095468-B-100 del Ministerio de Ciencia, Innovación y Universidades.

Marta Borrueco (iD https://orcid.org/0000-0007-7537-5673 es psicologa, Máster en psicología del deporte y de la actividad física e investigadora predoctoral en la Univerdidad Autónoma de Barcelona

Yago Ramis (D) https://orcid.org/0000-0003-3881-300X es psicólogo y Doctor en Psicología Universitat Autònoma de Barcelona.
Susana Pallarès (iD https://orcid.org/0000-0001-5793-9754 es psicóloga y Doctora en Psicología Universitat Autònoma de Barcelona.
Jaume Cruz (iD) https://orcid.org/0000-0001-6202-847X es psicólogo y Doctor en psicología Universitat Autònoma de Barcelona.

La correspondencia sobre este artículo debe enviar a la primera autora al Departament de Psicologia Bàsica, Evolutiva i de l'Educació, Facultat de Psicologia, Edifici B, Universitat Autònoma de Barcelona, 08193, Bellaterra (Cerdanyola del Vallès), Barcelona (España). E-mail: 
(e) monitoring of the program's effects through the time. The education program consisted of 5 sessions (i.e., one for each C) and the number of coaches attending to every workshop ranged from 6 to 12. Participants' satisfaction, assessed after every workshop, showed that coaches' expectations were fulfilled, and they considered that the program offered an appropriate setting to share experiences between them. However, coaches felt that more practical exercises were needed. This work can be used as a guide for sport psychology practitioners wanting to increase rigor by integrating scientific evidence into their applied practice, besides adding research evidence to the sport psychology field by publishing these experiences.

KEYWORDS: psychosocial development, evidence-based practice, coaching effectiveness, training program, positive youth development.

\section{Aplicação do modelo dos 5Cs para formação de treinadores: um exemplo de prática baseada em evidências}

RESUMO: O presente trabalho tem como objetivo desenvolver e implementar um programa de formação baseado em evidências, destinado a treinadores de um clube de hóquei em patins, a fim de otimizar o desenvolvimento psicossocial de seus atletas. Esta experiência profissional explica como, a partir de uma solicitação do clube, o Grupo de Estudos em Psicologia do Desporto e Atividade Física GEPE (Grup d'Estudis en Psicologia de l'Esport i de l'Activitat Física) desenvolveu um programa de formação adaptado às suas características e necessidades. Para desenvolver o programa, foram seguidas as fases propostas pelo Modelo EPE de PBE (ou seja, Prática Baseada em Evidências): (a) atualização documental por revisão de evidências científicas sobre a influência do treinador nos atletas, (b) análise de necessidades do clube e dos seus treinadores; (c) desenvolvimento e aplicação de um programa de formação baseado no modelo dos 5Cs (comprometimento, comunicação, concentração, controlo e confiança) para aumentar as capacidades dos treinadores de promover o desenvolvimento psicossocial de seus atletas; (d) avaliação do programa de formação e (e) monitorização dos efeitos do programa ao longo do tempo. 0 programa de formação consistiu em 5 sessões de formação teórico-práticas, uma para cada C, nas quais participaram de 6 a 12 treinadores por sessão. A satisfação dos participantes, avaliada após cada sessão, demonstrou que as sessões de formação responderam às expectativas dos treinadores e, além disso, estes consideraram que as sessões proporcionaram um contexto ideal para partilhar experiências com os seus colegas. Como áreas de melhoria, consideraram que deveriam ser adicionados mais conteúdos práticos. Este trabalho é apresentado como um exemplo da integração de evidências científicas na prática aplicada dos profissionais de psicologia do desporto, dando rigor à sua prática profissional.

PALAVRAS-CHAVE: desenvolvimento psicossocial, prática baseada em evidências, treinador/a, programa de formação, desenvolvimento juvenil positivo.

Artículo recibido: 07/04/2020 | Artículo aceptado: 29/05/2020

El aumento de la presencia de contenidos relacionados con la Psicología del Deporte (e.g., currículum académico en el grado de Psicología y en el de Ciencias del deporte, formación específica en cursos de entrenadores) en el contexto deportivo (De la Vega y Cremades, 2016), está promoviendo que cada vez sean más las instituciones y organizaciones deportivas que se interesan por el trabajo de aspectos psicológicos tanto por sus efectos en el rendimiento deportivo (Tod et al., 2017), como por los beneficios en el desarrollo integral de los deportistas (Côté y Hancock, 2014).

La Psicología del Deporte, según Weinberg y Gould (2019), tiene como objetivos entender (a) qué efectos tienen los factores psicológicos en la ejecución física y motora, y (b) qué efectos tiene la participación en el deporte en el desarrollo psicológico, la salud y el bienestar. Con el fin de llegar a esos objetivos, cada vez es más evidente la necesidad de incorporar la Práctica Basada en la Evidencia (PBE) en nuestra disciplina, ya que solamente así se logrará integrar la investigación y la práctica aplicada (Levant y Hasan, 2008), respondiendo de esta manera a las demandas de los usuarios mediante prácticas sustentadas en el conocimiento científco (e.g., Borrueco et al., 2019; Jordana et al., 2019).

El Grup d'Estudis de Psicologia de l'Esport i l'Activitat Física (Grupo de Estudios de Psicología del Deporte y de la Actividad Física; GEPE), con el fin de sistematizar y estructurar su servicio de consultoría, ha desarrollado el Modelo GEPE de PBE (i.e., Práctica Basada en la Evidencia; Ramis et al., 2019, ver figura 1), modelo que integra en la práctica aplicada la evidencia científica actual y relevante del ámbito de la Psicología del Deporte y que el GEPE utiliza para responder a las demandas externas de entidades deportivas. Este fue el caso reciente de un club de hockey sobre patines que contactó con el GEPE con el fin de incorporar a su proyecto deportivo acciones de formación en psicología dirigidas a sus entrenadores. El Hockey Club Sant Just (HCSJ) es un club de hockey sobre patines de más de 60 años de historia y localizado en Sant Just Desvern (Barcelona). El club cuenta actualmente con 23 equipos, tres de los cuales femeninos, que cubren todo el espectro de categorías de competición recogidas por la Federació Catalana de Patinatge (Federación 
Catalana de Patinaje; FCP), tienen equipos desde la categoría de prebenjamín hasta categorías absolutas (i.e., senior OK Liga Plata). En consecuencia, en su proyecto deportivo conviven secciones con objetivos más orientados al aprendizaje y desarrollo de habilidades (i.e., etapas de iniciación), con secciones donde los objetivos se focalizan en obtener resultados deportivos en contextos de competición (i.e., etapas de desarrollo de talento deportivo). El club contactó con el GEPE para formar a sus entrenadores/as encargados de las secciones de iniciación en los aspectos psicológicos relevantes para el contexto deportivo, ya que consideraban que la Psicología del Deporte podía ser un complemento útil para el desarrollo de su tarea y así ayudar a sus deportistas a gestionar las exigencias que se iban presentado a medida que se acercaban a un contexto centrado en la competición. A continuación, se detalla cómo, a partir de la recepción de la demanda, el GEPE desarrolló un proyecto de formación de entrenadores/as basado en el Modelo de las 5Cs (Harwood, 2008), modelo utilizado para trabajar los aspectos psicológicos en el contexto deportivo.

Figura 1. Modelo GEPE de Práctica Basada en la Evidencia (Ramis et al., 2019)

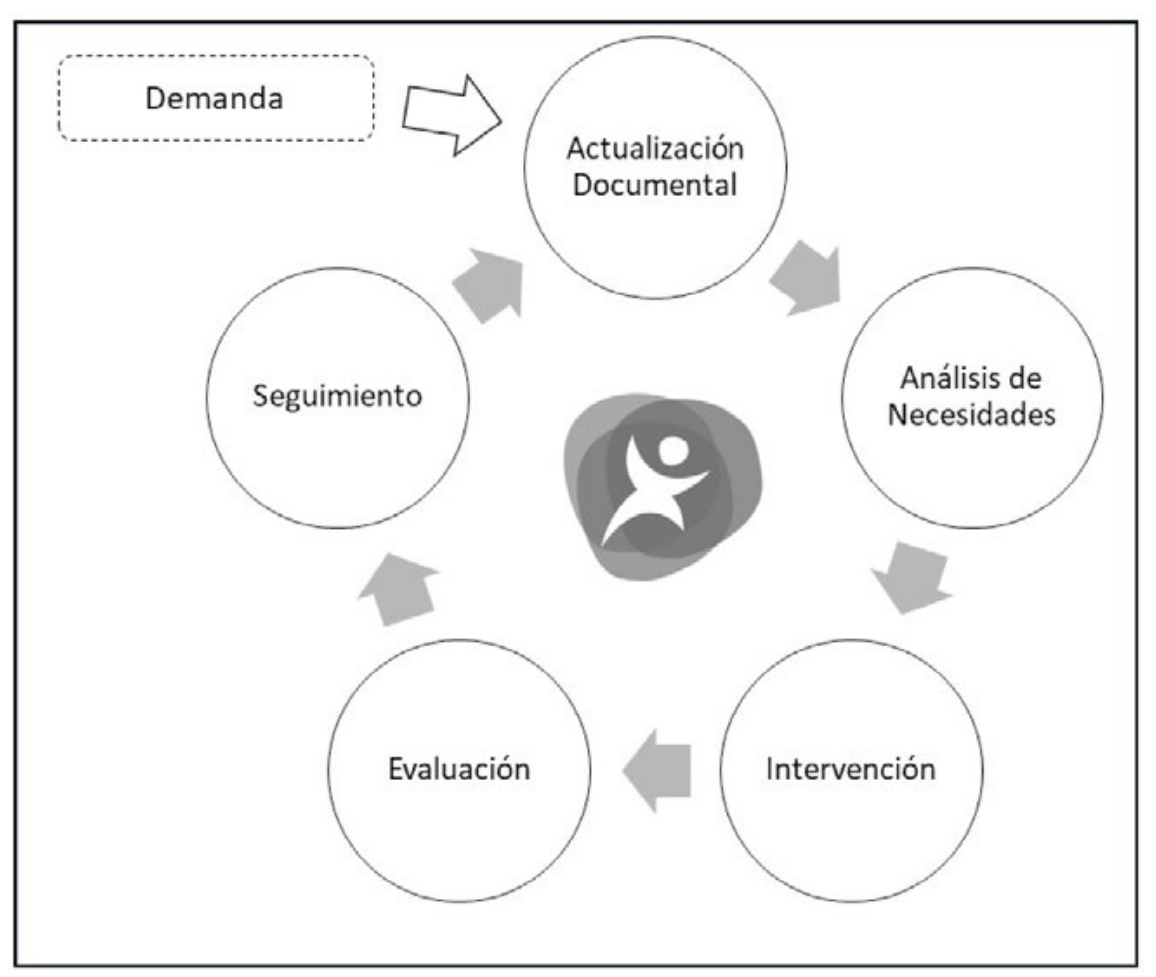

Nota. Reproducida con el permiso de los/las autores/as de "El Modelo GEPE de práctica basada en la evidencia: Integrando evidencia científica y práctica aplicada" de Ramis et al. (2019) en la Revista de Psicología Aplicada al Deporte y al Ejercicio Físico.

\section{Experiencia profesional}

Una vez se recibió la demanda, se concretó una reunión para decidir qué tipo de colaboración iba a ser más útil para abordarla, teniendo en cuenta las necesidades y recursos del club. Mediante la formalización de un convenio de cola- boración entre ambas instituciones se acordó (a) el tipo de acciones formativas que se llevarían a cabo (i.e., talleres teórico-prácticos) y (b) las condiciones del convenio (e.g., presupuesto, número de talleres, temporalidad).

Basándonos en las cinco fases del modelo GEPE de PBE, se acordaron las diferentes acciones que una de las psicólogas del deporte del GEPE llevaría a cabo para desarrollar el proyecto: (1) actualización documental a partir de la revisión de trabajos y publicaciones sobre la influencia del entrenador en los deportistas, (2) análisis de las necesidades de los agentes implicados mediante el desarrollo de un grupo de discusión con los entrenadores, una entrevista con un representante de la coordinación deportiva y uno de la dirección técnica del club, (3) intervención mediante el desarrollo e implementación de un programa de formación basado en talleres sobre las 5Cs para los entrenadores del club, (4) evaluación de la satisfacción y aprendizaje de los participantes en los talleres y, finalmente, (5) seguimiento de los efectos del programa de formación. A continuación, se detalla el procedimiento de cada una de estas fases del modelo, que sirvieron para darle forma esta experiencia profesional.

\section{Actualización documental}

Esta primera fase del modelo busca conocer qué fundamentos teóricos y qué procedimientos actuales son los más adecuados para abordar la demanda recibida. Concretamente para este proyecto, esta fase tenía el objetivo de explorar qué se estaba publicando en relación al trabajo de aspectos psicológicos a través del/la entrenador/a.

En los últimos años, las responsabilidades de esta figura han ido aumentando en número y complejidad, evolucionando desde responsabilizarse únicamente de los aspectos relacionados con las habilidades físicas, técnicas y tácticas necesarias para practicar el deporte en cuestión, hacia responsabilidades más globales y relacionadas con su influencia en el desarrollo holístico de los deportistas (Camiré et al., 2011). Esta evolución en número y en complejidad está relacionada a su vez con la concepción actual de que lo experimentado en el contexto deportivo (i.e., carrera deportiva) forma parte y contribuye en la vida de los deportistas a nivel general (Stambulova et al., 2020; Stambulova y Wylleman, 2014). Específicamente, el Modelo Holístico del Desarrollo de la Carrera Deportiva de Wylleman y Lavallee (2004; revisado posteriormente por Wylleman y Rosier, 2016; Wylleman, 2019), señalan cómo el desarrollo de la carrera deportiva se ve influido por las experiencias vividas en otras esferas (e.g., psicológica, psicosocial, académica-vocacional) de la vida de los deportistas. A su vez, según Côté y Hancock (2014) 
la práctica deportiva, sobre todo en edades de iniciación (i.e., infancia y adolescencia), se relaciona con (a) el mantenimiento de un estilo de vida activo, (b) el aprendizaje de habilidades derivadas del deporte, y (c) un desarrollo psicosocial óptimo. Por todo ello, es importante que el deportista en etapas de iniciación tenga experiencias positivas, disminuyendo así el riesgo de abandono del deporte (Holt y Knight, 2014; Somerset y Hoare, 2018).

El entorno en el que se desarrolla el deportista es uno de los factores más influyentes en la percepción de la experiencia deportiva (Mills y Pain, 2017). Henriksen et al. (2010), basándose en el Modelo Ecológico de Bronfenbrenner (1999, 2005), definen el entorno deportivo como un sistema dinámico de interrelaciones anidadas que engloba las relaciones más cercanas y directas del microsistema (e.g., club, entrenador/a, familia), las interrelaciones entre ellas, y un contexto más amplio a nivel de macrosistema donde se integran estas relaciones (e.g., comunidad, cultura). Desde este punto de vista, el entorno puede promover atributos internos clave (e.g., compromiso, competencia interpersonal, autoestima) para el desarrollo psicosocial de los deportistas a través de la influencia de los distintos agentes con los que se relacionan (Fraser-Thomas et al., 2005). De entre el conjunto de interrelaciones posibles dentro del entorno, la relación entrenador/a-deportista es una de las más estudiadas (Holt et al., 2016; Strachan et al., 2016).

Son numerosas las publicaciones basadas en detectar qué comportamientos debe realizar el/la entrenador/a para provocar consecuencias positivas en los deportistas (i.e., Coaching effectiveness; Horn 2008). Tradicionalmente, la investigación se ha centrado en evaluar la relación entre variables de forma cuantitativa, centrándose en examinar modelos relacionales y predictivos (e.g., Ramis et al., 2013; Cruz et al., 2011) y en la optimización de escalas y cuestionarios para deportes y poblaciones específicas (e.g., Newton et al., 2000; Ramis et al., 2010). Respecto a las intervenciones, en general han estado centradas en aspectos motivacionales, basándose en el conocimiento de que el ambiente (i.e., clima motivacional) generado por el/la entrenador/a tiene consecuencias en los deportistas en: (a) la calidad de motivación (e.g., Boixadós et al., 2004), (b) el compromiso deportivo (e.g., Torregrossa et al., 2011), (c) las muestras de fairplay y comportamientos prosociales (e.g., Borrueco et al., 2018; Kavussanu y Hodge 2018), o (d) el nivel de ansiedad competitiva (Ramis et al., 2017), entre otros.

Sin embargo, en algunos estudios e intervenciones tradicionales realizadas con entrenadores, no tenían en cuenta los diferentes escenarios del contexto de entrenamiento (i.e., pre-competición, entrenamiento, post-competición, competición), ni hacían referencia a situaciones específicas de cada deporte y, además, creían que no tenían recursos suficientes para incorporar el trabajo de habilidades psicosociales en sus sesiones (Harwood, 2016; Santos et al., 2019). Esta afirmación va en línea con la concepción de que para poder influir positivamente en sus deportistas es importante considerar la medida en que se ven capaces de influir en el aprendizaje y el rendimiento de sus deportistas (i.e., Coaching efficacy; Feltz et al.,1999), además de promover reflexiones sobre su filosofía y trabajar las habilidades necesarias para que puedan desarrollar estas estrategias (Santos et al., 2017).

Actualmente, una práctica aplicada basada en la evidencia que tiene en cuenta las diferentes situaciones y que está centrada en ofrecer pautas específicas para integrar las habilidades psicológicas en el contexto deportivo es el Modelo de las 5Cs de Harwood (Harwood, 2008; Harwood et al., 2015). Este modelo está a su vez enmarcado en la perspectiva del Desarrollo Positivo Juvenil (PYD; por sus siglas en inglés Positive Youth Development; Lerner, 2002, 2005), que defiende que los niños y adolescentes (e.g., deportistas en etapas de iniciación) tienen habilidades y recursos que deben potenciarse para así llegar a desarrollarse óptimamente (Lerner et al., 2015). El Modelo de las 5Cs se centra en aumentar la confianza y conocimiento de los/las entrenadores/as sobre su influencia en el desarrollo psicosocial de los deportistas (i.e., Coaching efficacy). Para ello, deben aprender estrategias para promover lo que se han denominado las $5 \mathrm{Cs}$ del desarrollo psicosocial: el compromiso, la comunicación, la concentración, el control y la confianza (Harwood, 2008).

\section{Análisis de necesidades}

En paralelo a la actualización documental y con el objetivo de conocer qué necesidades presentaba el club en general, y sus entrenadores/as en particular, y así poder ajustar e individualizar la propuesta al máximo, se llevaron a cabo (a) un grupo de discusión con entrenadores y, (b) una entrevista con la coordinación deportiva.

\section{Grupo de discusión con entrenadores}

Cinco entrenadores (i.e., E1, E2, E3, E4, E5) de entre 25 y 40 años $(M=26,5 ; D E=6,4)$, recomendados por la coordinación deportiva por su voluntad de colaborar y por su nivel de experiencia en el club (i.e., todos con más de cinco años de experiencia), participaron en el grupo de discusión que tenía el objetivo de explorar qué necesidades de formación presentaban. Para realizar el grupo de discusión se elaboró un guion semiestructurado que abarcaba: (a) el rol del psicólogo del deporte en un club de hockey, (b) la figura del entrena- 
dor en el deporte de iniciación, (c) la relación del entrenador con los otros agentes de influencia en el contexto deportivo (i.e., club, deportistas y familias), y (d) el conocimiento sobre las 5Cs del desarrollo óptimo. El grupo de discusión tuvo una duración de 90 minutos y fue grabado en audio para su posterior transcripción verbatim y análisis.

\section{Entrevista a la dirección técnica}

Además del grupo de discusión con entrenadores, se entrevistó al Coordinador Deportivo (CD; 39 años) y al Director Técnico (DT; 56 años) del club ya que conocían tanto la organización del club como la parte deportiva. Ambos con más de 20 años de experiencia en el deporte como jugadores y entrenadores. El primero lleva cuatro años en el club y realiza funciones de gestión de la parte deportiva (e.g., gestión de equipos, coordinación entrenadores/as), compaginando este rol con el de deportista del primer equipo del club. Por otro lado, el DT lleva más de cinco años en el club, y describe su función en términos de apoyo a la coordinación deportiva y a los entrenadores. Para realizar la entrevista se elaboró un guion de entrevista semi-estructurado similar al que se utilizó en el grupo de discusión con los entrenadores. En concreto, los temas a tratar fueron: (a) estructura del club, (b) figura del entrenador en el deporte de iniciación, (c) relación de la dirección deportiva con los otros agentes de influencia en el contexto deportivo (i.e., entrenadores, familias) y, (d) características del club (e.g., objetivos, fortalezas, necesidades detectadas). La entrevista duró 36 minutos y se grabó en audio, posteriormente se realizó una transcripción verbatim.

\section{Resultados del análisis de necesidades}

Pese a que se presentan conjuntamente, los datos recogidos fueron analizados por separado mediante un análisis temático deductivo (Braun y Clarke, 2006; Braun et al., 2016). Respecto a las necesidades de formación, la dirección técnica destacó la comunicación como el aspecto prioritario para el club, tal y como se puede observar en la siguiente reflexión realizada por el DT:

Me preocupa bastante la manera de comunicarse con los niños y el lenguaje... sobre todo que sean conscientes de cómo tienen que actuar en según qué situaciones. O sea, si tienes que dar un mensaje delante de cuatro jugadores o mejor de forma individual, si tienen que alzar el volumen de voz o no, [...] estas cosas de gestión del entrenamiento y de orientar un poco el mensaje, un poco cómo darlo y sobre todo la calidad del lenguaje.

La Tabla 1 tiene el objetivo de resumir las respuestas de los entrenadores respecto a las situaciones que considera-

\section{Tabla 1. Situaciones más demandantes detectadas por los entrenadores con relación a las 5Cs del desarrollo psicosocial de los deportistas}

\begin{tabular}{|c|c|c|}
\hline Comunicación & $\begin{array}{l}\text { Conocer las características de cada de- } \\
\text { portista para saber cómo comunicarse. }\end{array}$ & $\begin{array}{l}\text { "es importante saber cómo funciona el equipo y cómo es cada carácter. Cada persona } \\
\text { es diferente" (E4). }\end{array}$ \\
\hline Concentración & $\begin{array}{l}\text { Gestión de la falta de atención y con- } \\
\text { centración de los deportistas. }\end{array}$ & $\begin{array}{l}\text { "Existen muchas cosas que influencian mucho a los deportistas. Que si el móvil, que si } \\
\text { no sé qué... luego llegan aquí y están... están fuera" (E2). }\end{array}$ \\
\hline Control & $\begin{array}{l}\text { Gestión de situaciones estresantes } \\
\text { para los deportistas. }\end{array}$ & $\begin{array}{l}\text { "Juntas la edad, los estímulos que hemos hablado (e.g., móvil, videoconsola), a los pa- } \\
\text { dres y la competición y es que... se sabe de sobra que no saben gestionar esta presión y } \\
\text { pasa lo que pasa" (E1) } \\
\text { "Sienten rabia, frustración... la rabia de que no puede ser que las cosas no salgan y eso } \\
\text { tiene efecto en la pérdida de los nervios y enfadarse con el árbitro... en otros equipos } \\
\text { que son menos competitivos veo otras emociones" (E5). }\end{array}$ \\
\hline Compromiso & $\begin{array}{l}\text { Conseguir que los jugadores estén más } \\
\text { comprometidos con los entrenamien- } \\
\text { tos, competición y cultura del hockey. }\end{array}$ & $\begin{array}{l}\text { "las personas más comprometidas son las que hablan de hockey. Hablan de hockey y } \\
\text { después suelen ser las que vienen siempre a entrenar y que cuando pierden se ponen } \\
\text { objetivos más serios, que saben qué hicieron mal y qué tienen que cambiar" (E5). }\end{array}$ \\
\hline
\end{tabular}


ban más demandantes para cada una de las $5 \mathrm{Cs}$. Como se puede observar, los entrenadores son conscientes de que existen varios aspectos que se pueden trabajar para mejorar la experiencia de sus deportistas, detectando para cada uno de ellos (e.g., concentración) qué situaciones deben gestionar para su optimización (e.g., gestión de la falta de atención y concentración de los deportistas).

Además, respecto a la concepción de la figura del entrenador, tanto entrenadores como la dirección técnica coinciden en que las responsabilidades de los entrenadores van más allá de la gestión de los entrenamientos y competiciones. Ambos coinciden en que su labor e influencia excede lo puramente deportivo, por ejemplo, el E1 señala que como entrenador "les enseñas muchos valores", mientras que el E2, en la misma línea, opina que "también somos un poco educadores de estos chavales, ¿no? Me acuerdo de cuando yo tenía entrenador muchos de estos eran para mí unos referentes [...] les puedes ayudar, estás para ellos, y que trabajas para su bien también". Además, también se puede observar que se perciben como modelos de comportamiento. En este sentido, el E1 expone que "sí, un modelo a seguir. Y no nos olvidemos de la influencia que tiene esto, que es muchísima, no tanta como cuando tienen dieciocho, pero mucho más cuando son niños". Esta percepción del entrenador es compartida también por el DT, como se puede observar en el siguiente fragmento:

El mensaje que les hemos dado siempre es que ellos son formadores, pero no solamente de hockey [...] tienen muy claro que los chavales vienen a pasárselo bien, o sea, tienen que encontrar este puto en que los niños trabajen pero que también sea una actividad a la que vienen a divertirse.

Otro aspecto relevante a destacar son las relaciones que se establecen entre los diferentes agentes del club. Tanto los entrenadores como la dirección técnica coinciden en que existe una buena relación entre ellos ya que la dirección técnica respalda a los entrenadores, ofreciéndose sobre todo a resolver las posibles dudas y complicaciones que surjan. Este hecho se ve reflejado en la opinión del E1 y del E3, respectivamente:

Te puedes quedar bloqueado en una cosa. Pero hablas con CD y ya más o menos encuentras la solución [...] somos jóvenes, por mucho que nos guste y que podamos entender, a veces se nos escapan cosas y aquí es donde nos pueden reforzar personas con más experiencia como CD.

La implicación es brutal. Porque yo a CD le toco las narices [...] el otro día quedamos una hora antes y hablamos. Y nos reunimos en el bar, le comento de todos los partidos que tengo, le pregunto qué opina y él me da su feedback. Su implicación es muy grande.

El HCSJ apuesta por tener entrenadores que se hayan formado en el club, apostando por un modelo de juego propio y común en todos los equipos. Este hecho provoca que cuenten con pocos entrenadores y que muchos sean responsables de más de un equipo y, pese a que esto aumenta la carga de trabajo, tal y como destaca el CD:

Queremos que todos los entrenadores sean de aquí, del club por eso todos (los entrenadores) llevan más equipos de los que nos gustaría [...] después lo que sí que intentamos es que haya mucha cohesión de grupo [...] porque si tienes que sustituir que lo hagas de buen gusto. Intentamos hacer actividades como de cohesión de grupo [...] para incentivar que sean como un equipo, como un equipo de hockey pero un equipo de trabajo y con eso creo que hemos acertado porque todo el mundo funciona.

Pese a las dificultades, tanto la dirección técnica como los entrenadores destacan la buena relación que existe entre los propios entrenadores, apoyándose entre ellos cuando lo necesitan, tal y como se refleja en la opinión del E4:

Básicamente diría que todos los entrenadores hablamos mucho y también entre nosotros nos ayudamos con muchas cosas. Aquí tenemos un grupo de entrenadores que está bastante unido y que nosotros aparte de entrenadores, somos amigos. Y esto ayuda mucho a que funcione.

Además de la relación entre entrenadores entre sí y de los entrenadores con la dirección técnica, destaca también el papel de las familias en el entorno deportivo. Si bien no indican situaciones complicadas relevantes, es cierto que eventualmente tanto entrenadores como la dirección técnica tienen que lidiar con algunas quejas y problemáticas planteadas por las familias, sobre todo relacionadas con las decisiones de los entrenadores respecto a la repartición de minutos entre los deportistas, tal y como señala el E1:

Me decía qué tipo de trabajo había hecho y después venía y me decía cómo tenía que hacer las cosas y yo, obviamente, este tipo de conversaciones siempre las corto. Hay algunas cosas con las que estoy de acuerdo y otras que eran el trabajo en lo que yo creía o que yo había acordado con el coordinador o el director deportivo. Y había un tema especial, que no le gustaba un jugador del equipo y me lo vino a decir muy malamente. Que por qué le daba minutos.

Normalmente, los entrenadores se centran en intercambiar opiniones con las familias tras los entrenos o competiciones, sin la existencia de un protocolo de actuación específico, pero si surge alguna situación relevante o complicada, 
cuentan con el apoyo del CD, tal y como relata en el siguiente fragmento:

Intento no escabullirme, pero intento que, si tienen que hablar, que sea por cosas importantes, siempre estoy abierto por si viene cualquier padre, hablo de lo que sea, entonces así conoces los perfiles. Yo como conozco a todo el mundo ya sé por dónde vienen algunos... pero sí, yo con los padres tengo relación directa. Siempre que hay alguna cosa entre entrenador y padres, yo intento que, si lo pueden gestionar ellos que lo gestionen, pero si sobrepasa su diligencia entonces rápidamente pido que me lo deriven a mí.

Cabe destacar que, pese a que esta fase va conceptualmente después de la actualización documental, las acciones realizadas para formalizar el convenio de colaboración también se consideran parte del análisis de necesidades ya que es ahí donde se concreta la demanda y se delimita el tiempo y recursos que el club va a dedicar al proyecto.

\section{Intervención}

Una vez acordadas las condiciones de la colaboración, revisadas las investigaciones y prácticas más actuales, y analizadas las necesidades del club y de sus entrenadores, se elaboró una propuesta de trabajo con el objetivo de diseñar e implementar un programa de formación basado en la evidencia y dirigido a incrementar la habilidad y el conocimiento (i.e., coaching effectiveness) de los entrenadores encargados de las secciones de iniciación del club (i.e., desde prebenjamín hasta juvenil), para promover las 5Cs del desarrollo en sus sesiones de entrenamiento. Como se ha comentado anteriormente, las 5Cs propuestas por Harwood (2008), hacen referencia a cinco componentes considerados clave para el desarrollo psicosocial óptimo de los deportistas: (a) compromiso (i.e., calidad motivacional que guía el comportamiento de los deportistas durante entrenamientos y partidos), (b) comunicación (i.e., habilidad para relacionarse con los demás a través de cómo mandan y reciben información, (c) concentración (i.e., habilidad para focalizarse en el aspecto correcto en el momento correcto), (d) control (i.e., habilidad para regular los pensamientos, sentimientos y emociones para poder manejar el comportamiento en situaciones estresantes), y (e) confianza (i.e., creencia que tiene el deportista en el momento de ejecutar una habilidad según el nivel deseado).

Las acciones formativas propuestas seguían una tipología de taller interactivo, combinando contenidos teóricos relevantes con herramientas y recursos que los participantes pudieran utilizar en su día a día. En total se realizaron cinco sesiones (i.e., una para cada C), cada una con una duración aproximada de dos horas y repartidas a lo largo de aproximadamente 12 meses. Siguiendo las recomendaciones de Harwood y Steptoe (2017), todas las sesiones presentaban una estructura similar: (a) presentación de la C y debate inicial sobre qué harían para promocionarla, (b) principios teóricos claves y características que sustentan cada $\mathrm{C}$, (c) ventajas de desarrollar la C y, (d) presentación y debate sobre estrategias y pautas que pueden facilitar el desarrollo de la $\mathrm{C}$ en el entrenamiento. A partir del segundo taller, al inicio de cada sesión se dedicaba un tiempo a que los entrenadores explicaran si habían puesto en práctica algún aspecto aprendido hasta ese momento.

La Tabla 2 presenta el orden y un resumen del contenido trabajado en cada uno de los talleres. A modo de ejemplo, el taller enfocado a la promoción del compromiso tenía como objetivos (a) entender qué impacto tenía el entrenador en la motivación de sus deportistas, y (b) aprender pautas y estrategias para aplicar estos conocimientos a las sesiones de entrenamientos y/o competiciones. Para ello, se empezó preguntando a los entrenadores qué entendían ellos por motivación y se hizo un debate sobre la motivación de sus deportistas (e.g., cómo saben si están motivados, qué les motiva). A continuación, se explicaron brevemente los principios teóricos claves para favorecer el compromiso de los deportistas (i.e., motivación autodeterminada, necesidades psicológicas básicas, clima motivacional autonomía/controlador y tarea/resultado) y las ventajas derivadas de tener jugadores comprometidos. Finalmente, se ofrecieron estrategias y pautas para incorporar el trabajo del compromiso en las sesiones de entrenamiento (e.g., argumentar por qué se realizan las actividades, fomento de la autonomía, apertura a la crítica y a los sentimientos negativos).

\section{Evaluación}

Esta fase tuvo el objetivo de valorar la propuesta de formación planteada. Si bien es cierto que la evaluación del programa es continua desde el momento de su diseño, ya que desde que la recepción de la demanda estuvimos en contacto continuo con el club para perfilar las acciones a desarrollar, también es necesario evaluar si las acciones están teniendo los efectos esperados (Ramis et al., 2019). Siguiendo los criterios básicos (Fernández-Ballesteros, 2001; Kirkpatrick y Kirkpatrick, 2006), existen cuatro niveles de valoración: (a) reacción, (b) aprendizaje de nuevos conocimientos, (c) implementación de los aprendizajes y, (d) efecto final de la intervención en el entorno. Sin embar- 
Tabla 2. Información sobre las sesiones realizadas, sus objetivos y ejemplos de contenidos trabajados

\begin{tabular}{|c|c|c|c|}
\hline Sesión & Objetivo & Ejemplos de estrategias & Comportamientos esperados en los deportistas \\
\hline Compromiso & $\begin{array}{l}\text { Motivación y estrategias para } \\
\text { fomentar la autonomía de los } \\
\text { deportistas. }\end{array}$ & $\begin{array}{l}\text { Promoción de la autonomía y toma de } \\
\text { decisiones. } \\
\text { Facilitar información relevante. }\end{array}$ & $\begin{array}{l}\text { Motivación autodeterminada. } \\
\text { Objetivos orientados a la tarea/resultado. }\end{array}$ \\
\hline Comunicación & $\begin{array}{l}\text { Estilo de comunicación } \\
\text { efectivo. }\end{array}$ & $\begin{array}{l}\text { Instrucciones directas y claras. } \\
\text { Refuerzo del esfuerzo, más allá del } \\
\text { resultado de la acción. }\end{array}$ & $\begin{array}{l}\text { Ofrecer feedback y escuchar activamente. } \\
\text { Esfuerzo y persistencia. }\end{array}$ \\
\hline Concentración & $\begin{array}{l}\text { Aspectos atencionales y } \\
\text { gestión del error. }\end{array}$ & $\begin{array}{l}\text { Gestión activa de los errores. } \\
\text { Refuerzo a deportistas que están aten- } \\
\text { tos en el momento adecuado. }\end{array}$ & $\begin{array}{l}\text { Focalización en los aspectos relevantes para la } \\
\text { tarea a desarrollar, evitando distractores. } \\
\text { Capacidad de cambiar el foco de atención. }\end{array}$ \\
\hline Control & $\begin{array}{l}\text { Control de la activación y } \\
\text { estrategias de afrontamiento } \\
\text { en situaciones estresantes. }\end{array}$ & $\begin{array}{l}\text { Detección de creencias desadaptativas. } \\
\text { Elaborar una rutina pre, durante, y post } \\
\text { competición. }\end{array}$ & $\begin{array}{l}\text { Detección de aquello que se puede (o no) controlar. } \\
\text { Utilizar estrategias de afrontamiento de situaciones } \\
\text { estresantes (e.g., orientadas a la tarea, orientadas a } \\
\text { la emoción, orientadas a la evitación). }\end{array}$ \\
\hline Confianza & $\begin{array}{l}\text { Establecimiento de objetivos } \\
\text { y promoción de la percepción } \\
\text { de competencia. }\end{array}$ & $\begin{array}{l}\text { Animar a la persistencia y a establecer } \\
\text { nuevos retos. } \\
\text { Generar situaciones donde todos } \\
\text { puedas aprender nuevas habilidades, } \\
\text { independientemente del nivel deportivo. }\end{array}$ & $\begin{array}{l}\text { Objetivos realistas pero que supongan un reto. } \\
\text { Internalización de los cumplidos. }\end{array}$ \\
\hline
\end{tabular}

Nota. Adaptada de "Developing Consulting in a Professional Football Academy: The 5Cs Coaching Efficacy Program" por C. G. Harwood, 2008, The Sport Psychologist.

go, en este proyecto, debido a las condiciones del convenio, solamente se evaluaron los dos primeros (i.e., reacción, aprendizaje de nuevos conocimientos), que coinciden con los aspectos que se pueden evaluar durante el programa. En total, entre 6 y 12 entrenadores (i.e., 11 entrenadores y una entrenadora) con un rango de edad de 18 a 51 años ( $M$ = 27.1; $D E=10.8)$, participaron en cada taller. A continuación, se describe su reacción y sus aprendizajes tras asistir a los talleres.

\section{Evaluación de la satisfacción}

Para medir la reacción de los entrenadores se diseñó una encuesta de satisfacción ad-hoc que se administró tras finalizar cada taller. La encuesta constaba de dos partes: (a) una valoración general del taller (i,e., percepción de aprendizaje, aplicabilidad de los contenidos, satisfacción general, cumplimiento de expectativas, material y contenidos, dinámica establecida) mediante nueve ítems con una escala de respuesta de tipo Likert ( 1 = Nada a $5=$ Mucho) y, (b) una pregunta de respuesta abierta sobre los aspectos mejor y peor valo- rados del taller. En líneas generales, los resultados muestran una valoración positiva (i.e., puntuaciones superiores a 4) de prácticamente todos los aspectos, destacando entre ellos la posibilidad de compartir experiencias a partir de la dinámica generada $(M=4.8 ; D E=0.56)$ y la aplicabilidad de los contenidos utilizados $(M=4.6 ; D E=0.63)$. Además, en general se mostraron satisfechos con los talleres $(M=4.5 ; D E=0.52)$ y consideraron que los talleres habían satisfecho sus expectativas iniciales $(M=4.5 ; D E=0.52)$.

Con relación a la valoración cualitativa, los participantes expresaron y valoraron positivamente la oportunidad de crear debate y la posibilidad de poder compartir experiencias (e.g., "clima para poder hablar con comodidad", "el debate que se crea es enriquecedor), así como el contenido práctico y aplicable de cada taller (e.g., "contenido muy adecuado y práctico, ideas esquemáticas con ejemplos para clarificar las ideas", "me parece muy positivo tratar aspectos reales de los entrenadores"). En cambio, los aspectos a mejorar fueron el horario en que se realizaban los talleres (i.e., de 19 a $21 \mathrm{~h}$ ) y la falta de más actividades prácticas (e.g., "incluir algún role playing", "más tiempo de debate", "ejemplos más prácticos"). 


\section{Evaluación de los aprendizajes}

Además de evaluar la satisfacción de los participantes, se realizó una valoración sobre el conocimiento adquirido por los entrenadores tras finalizar cada taller (i.e., "concretamente, ¿qué mensajes o ideas te llevas a casa?"). Después de analizar las respuestas de los participantes, se puede observar como estos talleres fueron útiles para aprender conceptos referentes a la evidencia científica que sustentaba cada sesión. Por ejemplo, los participantes hacen referencia a: (a) el estilo de comunicación (e.g., "la importancia de la buena comunicación con los deportistas, y tener empatía en las diferentes situaciones que se puedan dar, tanto en partidos como en entrenamientos"), (b) el clima motivacional (e.g., "es importante crear un buen ambiente entre los jugadores, $y$ entre jugador-entrenador"), y (c) la concentración y gestión de errores (e.g., "mensaje de autogestión de errores", "rutinas pre-partido, durante el partido y post-partido). Además, también reflexionan sobre el aprendizaje de pautas y estrategias específicas del trabajo de cada C, por ejemplo, respecto a la comunicación comentaban que "no siempre tienes la razón, tienes que escuchar a tus jugadores, igual que ellos lo hacen contigo", "debes saber cuándo es un buen momento y cuándo no para animar, reforzar y/o reconocer la acción de tu deportista".

\section{Seguimiento}

La última fase del modelo, la de seguimiento, hace referencia a la necesidad de evaluar si las acciones desarrolladas tienen continuidad en el tiempo. Es decir, tiene el objetivo de reevaluar los elementos de implementación y los efectos finales en un momento más distante de la intervención. En este caso, se pretendía comprobar si los entrenadores conservan las pautas y estrategias aprendidas en los talleres y si han sido capaces de integrarlas en las sesiones de entrenamiento. Al finalizar las sesiones se contactó con el responsable del proyecto en el club (i.e., CD) para valorar el impacto que estaban teniendo los talleres en el club. Siendo conscientes que, al no poder trabajar en la pista de juego, era complicado valorar si la formación estaba teniendo efectos reales en los deportistas. Sin embargo, el CD señaló que el hecho de estar llevando a cabo acciones de este tipo sí era útil para reforzar la relación entre los entrenadores y reforzar además la percepción de apoyo que tenían del club, además de ser útil porque los entrenadores adquirían recursos para gestionar situaciones (e.g., gestión de errores, estilo de comunicación).

\section{Discusión}

El presente trabajo ofrece la descripción de la implementación de talleres formativos basados en la evidencia en un club de hockey sobre patines. Esta experiencia profesional se enmarca en el programa de consultoría del GEPE, el cual se basa en el Modelo GEPE de PBE (Ramis et al., 2019). Mediante la revisión de evidencia científica actual sobre investigaciones y procedimientos relacionados con la influencia del entrenador en los deportistas, y considerando las necesidades de los entrenadores y de la propia organización (i.e., dirección técnica), se diseñó e implementó un programa de formación para entrenadores con el doble objetivo de (a) concienciar sobre su capacidad de influir en los deportistas, y (b) ofrecer pautas y estrategias para integrar las 5Cs del desarrollo en las sesiones de entrenamiento (i.e., compromiso, comunicación, concentración, control y confianza).

Esta propuesta nace de la tendencia, cada vez más presente en nuestro ámbito, de adaptar las intervenciones a las experiencias de los participantes (e.g., Cruz et al., 2016) y a las demandas de la propia organización deportiva (Santos et al., 2019), teniendo en cuenta los recursos y necesidades de ambos (Ramis et al., 2019). Además, se sigue la recomendación de Camiré y Santos (2019) de hacer explícitas las situaciones y comportamientos relacionados con la promoción del desarrollo psicosocial de los deportistas, apostando por el aprendizaje de habilidades y estrategias que podrán ser útiles para otros contextos de la vida. Este trabajo, por tanto, da un paso en la dirección adecuada para estudiar y ejemplificar cómo el deporte puede influir en el desarrollo de los deportistas (Jones et al., 2016).

Además, en la fase de análisis de necesidades se observó que los entrenadores percibían dificultades a la hora de interactuar con las familias debido a las exigencias competitivas de éstas. Estos resultados van en línea con lo encontrado por Holt et al. (2016) ya que, más allá del entrenador, existen otros agentes del contexto deportivo que influyen en la promoción del desarrollo psicosocial de los deportistas (i.e., familias, organización y compañeros). Además, Harwood et al. (2019) y Strachan et al. (2011) encontraron que implicar a las familias en el proceso de formación era clave para que los programas de promoción del desarrollo de los deportistas tuvieran éxito.

\section{Limitaciones y futuras investigaciones}

Se debe tener en cuenta que en un servicio de consultoría quien marca las condiciones de la colaboración es el cliente (e.g., organización, club deportivo, federación) y, a veces, las 
necesidades de los distintos agentes implicados no pueden ser abordadas con toda la profundidad deseada debido a la falta de recursos (e.g., presupuesto, instalaciones, gestión del tiempo). Por ejemplo, el desarrollo del Modelo de las 5Cs está concebido para trabajarse durante una temporada y con personal psicólogo a tiempo completo en el club (Harwood y Anderson, 2015). Además de una primera fase de formación, en el trabajo de las 5Cs con entrenadores se contempla una fase de progresión que tiene el objetivo aplicar lo aprendido en las sesiones de entrenamiento. En este mismo sentido, si bien se ha podido evaluar la reacción y el aprendizaje de los entrenadores tras los talleres (i.e., fase de evaluación), al no realizarse la fase de progresión, no se ha podido evaluar la transferencia de los contenidos a la práctica, ni el efecto de éstos en el desarrollo psicosocial de sus deportistas, así como tampoco se han registrado las percepciones y experiencias de los deportistas. Además, un diseño longitudinal también sería útil para conocer en qué momento aparecen las dificultades y cómo influyen en experiencia y práctica de los entrenadores (Santos et al., 2019).

Como hemos comentado anteriormente, existe numerosa literatura sobre la relación entrenador-deportista (Holt et al., 2016; Strachan et al., 2016). Sin embargo, según Harwood (2016), hay poca evidencia sobre intervenciones basadas en la evidencia en distintos deportes y que consideren las diferentes demandas y roles de los entrenadores según el tipo de entorno (e.g., desarrollo del talento) y el momento de la etapa deportiva en la que se encuentran (e.g., iniciación, profesional, élite). Si bien este trabajo aporta evidencia sobre un contexto específico (i.e., hockey sobre patines y deportistas en etapa de iniciación o amateurs), está centrada únicamente en la influencia del entrenador en situaciones de rendimiento deportivo. El GEPE tiene una larga tradición trabajando con la figura del entrenador. Desde que en los años 80 empezara con la evaluación de su comportamiento durante las competiciones (e.g., Cruz et al., 1987), sus trabajos han ido evolucionando hacia intervenciones más individualizadas (e.g., Sousa et al., 2006) y centradas en su bienestar (e.g., Alcaraz et al., 2015). Recientemente, las investigaciones se han centrado en explorar el rol del entrenador en diversas situaciones "fuera del terreno/pista" con el fin de entender qué influencia juega en la transición de junior a senior y en la retirada deportiva (e.g., Jordana et al., 2017; Torregrossa et al., 2016). Por todo ello, es importante que futuras investigaciones diferencien entre ambos tipos de situaciones para tener una concepción más global de la influencia del entrenador.

Finalmente, en este trabajo se ha utilizado el género masculino para referirse a las 12 personas que participaron en los talleres, ya que solamente había una mujer entrenadora en el club. A pesar de que el club cuenta con varios equipos femeninos, solamente hay una entrenadora. Esta diferencia entre el número de entrenadores y entrenadoras es algo común en el contexto deportivo (Hovden y Tjønndal, 2019). Además, el conocimiento generado tanto desde la Psicología del Deporte en general, como de la investigación de la figura del entrenador en particular, han sido construidos tradicionalmente desde la perspectiva masculina y desde una epistemología positivista (Cooky, 2016; Norman y Rankin-Wright, 2018). Por esta razón, es importante que futuras investigaciones exploren cómo las entrenadoras construyen y experiencian su rol, teniendo en cuenta los diferentes mecanismos y estructuras de poder (i.e., interseccionalidad) y la influencia específica de la cultura en la que se encuentran inmersas (Ryba, 2017; Ryba et al., 2010).

\section{Aplicaciones practicas}

El Modelo GEPE de PBE apuesta por integrar la evidencia científica más actual a nuestra práctica profesional en el ámbito de la Psicología del Deporte. Esta propuesta permite acercar posturas entre aquellos que consideran que una u otra debe prevalecer y guiar el desarrollo de nuestra disciplina. Es importante entender que basar nuestros procedimientos en documentación científica actual y de calidad, aporta rigor a nuestra práctica profesional. Además, también es importante recalcar que el Modelo GEPE de PBE es sostenible siempre y cuando los grupos y personas del ámbito de la Psicología del Deporte divulguen el conocimiento generado tras sus investigaciones y/o prácticas aplicadas. Esto permitirá que futuros profesionales puedan integrar el conocimiento de anteriores experiencias (i.e., actualización documental), y así ir sumando el conocimiento de todos para mejorar el ámbito profesional.

Por último, este trabajo aporta evidencia sobre cómo puede optimizarse la influencia del/la entrenador/a en el desarrollo psicosocial de sus deportistas. En concreto, se presenta un programa de formación dirigido a entrenadores/as que pretende aumentar sus habilidades y conocimientos sobre el trabajo de aspectos psicológicos en las sesiones de entrenamiento, sirviendo así de ejemplo para otros/as profesionales del ámbito que tengan objetivos similares.

\section{Referencias}

Alcaraz, S., Viladrich, C., Torregrosa, M. y Ramis, Y. (2015). Club and Players' Pressures on the Motivation, Vitality and Stress of Development Coaches. International Journal of Sports Science and Coaching, 10(2), 365-378. https://doi.org/10.1260\% 2F1747-9541.10.2-3.365 
Braun, V. y Clarke, V. (2006). Using thematic analysis in psychology. Qualitative Research in Psychology, 3(2), 77-101. https://doi.org/10.1191/1478088706ap063oa

Braun, V., Clarke, V. y Weate. P. (2016). Using thematic analysis in sportand exerciseresearch. En B. Smithy A. Sparkes (Eds.), International handbook on qualitative research in sport and exercise (pp. 191-205). Routledge. https://doi.org/10.4324/9781315762012

Bronfenbrenner, U. (1999). Environments in developmental perspective: Theoretical and operational models. En S. L. Friedman., y T. D. Wachs (Eds.), Measuring environment across the life span. Emerging methods and concepts (pp. 2-28). American Psychological Press.

Bronfenbrenner, U. (2005). The bioecological theory of human development. En U. Bronfenbrenner (Ed.), Making Human Beings Human: Bioecological Perspectives on Human Development (pp. 3-15). Sage.

Borrueco, M., Alcaraz, S., Ramis, Y. y Cruz, J. (2019). "No es solo pitar": Diseño e implementación de un taller de formación para árbitros y jueces de deporte escolar. Revista de Psicología Aplicada al Deporte y la Educación Física, 4, e12.

https://doi.org/70.5093/rpadef2019a11

Borrueco, M., Angulo-Brunet, A., Viladrich, C., Pallarès, S. y Cruz, J. (2018). Relationship between motivational climate, sportspersonship and disposition to cheating in young soccer players. Revista de Psicologia del Deporte, 27(3), 13-20.

Boixadós, M., Cruz, J., Torregrossa, M. y Valiente, L. (2004). Relationships among motivational climate, satisfaction, perceived ability, and fair play attitudes in young soccer players. Journal of Applied Sport Psychology, 16(4), 301-317. https://doi. org/10.1080/10413200490517977

Camiré, M., Forneris, T., Trudel, P. y Bernard, D. (2011). Strategies for helping coaches

facilitate positive youth development through sport. Journal of Sport Psychology in Action, 2(2), 92-99. https://doi.org/10.108 0/21520704.2011.584246

Camiré, M. y Santos, F. (2019). Promoting Positive Youth Development and Life Skills in Youth Sport: Challenges and Opportunities amidst Increased Professionalization. Journal of Sport Pedagogy and Research, 5(1), 27-34. https://doi.org/10.1080/21 520704.2011.584246

Cooky, C (2016). Feminisms. En B. Smith y A. Sparkes, Routdlege Handbook of Qualitative Research in Sport and Exercise (pp. 7587). Routdlege.

Côté, J. y Hancock, D. J. (2014). Evidence-based policies for youth sport programmes. International Journal of Sport Policy and Politics, 8(1), 51-65. https://doi.org/10.1080/19406940.2014.9 19338

Cruz, J., Bou, A., Ferrández, J. M., Martin, M., Monrás, J., Monfort, N. y Ruiz, A. (1987). Avaluació conductual de les interaccions entre entrenadors i jugadors de bàsquet escolar. Apunts Medicina de l'Esport, 24(092), 89-98.

Cruz, J., Mora, A., Sousa, C., y Alcaraz, S. (2016). Effects of an Individualized Programo $n$ Coaches' Observed and Perceived Behavior. Revista de Psicología del Deporte, 25(1), 137-144.

Cruz, J., Torregrossa., M. Sousa, C., Mora, A. y Viladrich, C. (2011). Efectos conductuales de programas personalizados de asesoramiento a entrenadores en estilo de comunicación y clima motivacional. Revista de Psicología del Deporte, 20(1), 179-195.
De la Vega, R. y Cremades, G. (2016). The development of sport psychology in Spain: The role played by sport institutions. International Journal of Sport and Exercise Psychology, 16(1), 65-76. https://doi.org/10.1080/1612197X.2016.1154091

Fernández-Ballesteros, R. (2001). Evaluación de programas: una guía práctica en ámbitos sociales, educativos y de la salud. Síntesis.

Fraser-Thomas, J., Côté, J. y Deakin, J. (2005). Youth sport programs: An avenue to foster positive youth development. Physical Education and Sport Pedagogy, 10(1), 19-40. https://doi.org/10.1080/1740898042000334890

Feltz, D. L., Chase, M. A., Moritz, S. A. y Sullivan. P. J. (1999). A conceptual model of coaching efficacy: Preliminary investigation and instrument development. Journal of Educational Psychology, 91(4), 765-776. https://doi.org/10.1037/0022-0663.91.4.765

Harwood, C. G. (2008). Developmental Consulting in a Professional Football Academy: The 5Cs Coaching Efficacy Program. The Sport Psychologist, 22(1), 109-133. https://doi.org/10.1123/ tsp.22.1.109

Harwood, C. G. (2016). Enhancing coaching efficacy in the psychosocial development of athletes. En R. Thelwell, C. G. Harwood y I. Greenlees (Eds.), The psychology of sports coaching (pp. 203217). Routledge.

Harwood, C. G. y Anderson, R. (2015). Coaching psychological skills in youth football: Developing the 5Cs. Bennion Kearny Limited.

Harwood, C. G., Barker, J. B. y Anderson, R. (2015). Psychosocial Development in Youth Soccer Players: Assessing the Effectiveness of the 5Cs Intervention Program. The Sport Psychologist, 29(4), 319-334. https://doi.org/10.1123/tsp. 2014-0161

Harwood, C. G., Knight, C. J., Thrower, S. M. y Berrow, S. R. (2019). Advancing the study of parental involvement to optimise the psychosocial development and experiences of young athletes. Psychology of Sport and Exercise, 42, 66-73. https://doi.org/10.1016/i.psychsport.2019.01.007

Harwood, C. G. y Steptoe, K. (2017). Excellence together: Integrated sport psychology for coaches and young athletes. In C. J. Knight, C. G. Harwood y D. Gould (Eds.), Sport psychology for young athletes (pp. 288-302). Routledge.

Henriksen, K., Stambulova, N. y Roessler, K. K. (2010). Successful talent development in track and field: Considering the role of environment. Scandinavian Journal of Medicine and Science in Sports, 20(2), 122-132. https://doi.org/10.1111/j.16000838.2010.01187.X

Holt, N. L. y Knight, C. J. (2014). Participation in youth sport. En N. L. Holt y C. J. Knight (Eds.), Parenting in youth sport: From research to practice (pp. 16-27). Routledge.

Holt, N. L., Neely, K. C., Slater, L. G., Camiré, M., Côté, J., Fraser-Thomas, J., MacDonald, D., Strachan, L. y Tamminen, K. A. (2016). A grounded theory of positive youth development through sport based on results from a qualitative meta-study. International Review of Sport and Exercise Psychology, 10(1), 1-49. https://doi.org/10.1080/1750984X.2016.1180704

Horn, T. S. (2008). Coaching effectiveness in the sport domain. En T. S. Horn (Ed.), Advances in sport psychology (pp. 239-267, 455459). Human Kinetics.

Hovden, J. y Tjønndal, A. (2019). The gendering of coaching from an athlete perspective: The case of Norwegian boxing. Interna- 
tional Review for the Sociology of Sport, 54(2), 239-255.

https://doi.org/10.1177/1012690217715641

Jones, G. J., Edwards, M. B., Bocarro, J. N., Bunds, K. S., y Smith, J. W. (2016). An integrative review of sport-based youth development literature. Sport in Society, 20(1), 161-179. https://doi.org/ 10.1080/17430437.2015.1124569

Jordana, A., Pons, J., Borrueco, M., Pallarès, S. y Torregrossa, M. (2019). Implementación de un Programa de Asistencia de Carrera Dual en un club multideportivo privado. Revista de Psicología Aplicada al Deporte y la Educación Física, 4, e9. https://doi.org/10.5093/rpadef2019a8

Jordana, A., Torregrossa, M., Ramis Laloux, Y. y Latinjak, A. T. (2017). Retirada del deporte de élite: Una revisión sistemática de estudios cualitativos. Revista de Psicología del Deporte, 2017, 26(4), 68-74

Kavussanu, M. y Hodge, K. (2018). The coach's role on moral behaviour in sport. En R. Thelwell., y M. Dicks (Eds.), Professional advances in sports coaching: research and practice. Routledge

Kirkpatrick, D. L. y Kirkpatrick, J. D. (2006). Evaluating training programs: The four levels. (3rd ed.). Berrett-Koehler.

Lerner, R. M. (2002). Concepts and theories of human development (3rd ed.). Lawrence Erlbaum Associates Publishers.

Lerner, R. M. (2005, September). Promoting positive youth development: Theoretical and empirical bases. White paper prepared for the Workshop on the Science of Adolescent Health and Development, National Research Council/Institute of Medicine. National Academies of Science.

Lerner, R. M., Lerner, J. V., Bowers, E. P. y Geldhof, G. J. (2015). Positive Youth Development and Relational-Developmental-Systems. En. R. M. Lerner (Ed.), Handbook of Child Psychology and Developmental Science (7th ed., pp. 1-38). John Wiley \& Sons, INC.

Levant, R. F. y Hasan, N. T. (2008). Evidence-based practice in psychology. Professional Psychology: Research and Practice, 39(6), 658-662. https://doi.org/70.1037/0735-7028.39.6.658

Mills, A. y Pain, M. (2017). Creating Effective Development Environments for the Adolescent Athlete. En R. Thelwell, C. Harwood. y I. Greenlees (Eds.) The Psychology of Sports Coaching (pp. 2137). Routdlege.

Newton, M., Duda, J. L. y Yin, Z. (2000). Examination of the psychometric properties of the Perceived Motivational Climate in Sport Questionnaire-2 in a sample of female athletes. Journal of Sports Sciences, 18(4), 275-290.

https://doi.org/10.1080/026404100365018

Norman, L. y Rankin-Wright, A. (2018). Surviving rather than thriving: Understanding the experiences of women coaches using a theory of gendered social well-being. International Review for the Sociology of Sport, 53(4), 424-540.

https://doi.org/10.1177/1012690216660283

Ramis, Y., Torregrossa, M., Pallarés, S., Viladrich, C. y Cruz, J. (2019). El modelo GEPE de práctica basada en la evidencia: Integrando la evidencia científica en la práctica aplicada. Revista de Psicología Aplicada al Deporte y al Ejercicio Físico, 4(2), Artículo e13 https://doi.org/10.5093/rpadef2019a12

Ramis, Y., Torregrossa, M., Viladrich, C. y Cruz, J. (2010). Adaptación y validación de la versión española de la Escala de Ansiedad Competitiva SAS-2 para deportistas de iniciación. Psicothema, 22(4), 1004-1009.
Ramis, Y., Torregrossa, M., Viladrich, C. y Cruz, J. (2013). El apoyo a la autonomía generado por entrenadores, compañeros y padres y su efecto sobre la motivación autodeterminada de deportistas de iniciación. Anales de Psicología, 29(1), 243-248. http://dx.doi.org/10.6018/analesps.29.1.124011

Ramis, Y., Torregrossa, M., Viladrich, C. y Cruz, J. (2017). The effect of coaches' controlling style on the competitive anxiety of young athletes. Frontiers in psychology, 8, 572. https://doi.org/10.3389\%2Ffpsyg.2017.00572

Ryba, T. V. (2017). Cultural sport psychology: a critical review of empirical advances. Current Opinion in Psychology, 16, 123-127. https://doi.org/10.1016/i.copsyc.2017.05.003

Ryba, T. V., Schinke, R. J. y Tenenbaum, G. (Eds.). (2010). The cultural turn in sport psychology. Fitness Information Technology.

Santos, F., Camiré, M., MacDonald, D., Campos, H., Conceição, M. y Silva, A. (2017). Youth sport coaches' perspective on positive youth development and its worth in mainstream coach education courses. International Sport Coaching Journal, 4(1), 38-46. https://doi.org/10.1123/iscj.2016-0092

Santos, F., Gould, D. y Strachan, L. (2019). Research on Positive Youth Development-focused Coach Education Programs: Future Pathways and Applications. International Sport Coaching Journal, 6(1), 132-138. https://doi.org/10.1123/iscj.2018-0013

Somerset, S. y Hoare, D. J. (2018). Barriers to voluntary participation in sport for children: A systematic review. BMC Pediatrics, 18(1). https://doi.org/10.1186/s12887-018-1014-1

Sousa, C., Cruz, J., Torregrosa, M., Vilches, D. y Viladrich, C. (2006). Evaluación conductual y programa de asesoramiento personalizado a entrenadores (PAPE) de deportistas jóvenes. Revista de Psicología del Deporte, 15(2), 263-278.

Stambulova, N., Ryba, T. y Henriksen, K. (2020). Career development and transitions of athletes: The international society of sport psychology position stand revisited. International Journal of Sport and Exercise Psychology. Publicación avance online. https://doi.org/10.1080/1612197X.2020.1737836

Stambulova, N. y Wylleman, P. (2014). Athletes' career development and transitions. En A. G. Papaioannou, D. Hackfort (Eds.). Routledge companion to sport and exercise psychology (pp. 629644). Routledge.

Strachan, L., Côté, J. y Deakin, J. (2011). A new view: Exploring positive youth development in elite sport contexts. Qualitative Research in Sport, Exercise and Health, 3(1), 9-32. https://doi.org/10.1080/19398441.2010.541483

Strachan, L., Fraser-Thomas y Nelson-Ferguson, K., (2016). An Ecological perspective on High Performance Sport and Positive Youth Development. En N. L. Holt (Ed.), Positive Youth Development Through Sport (pp. 57-68). Routledge.

Tod, D., Hutter, R. y Eubank, M. (2017). Professional development for sport psychology practice. Current Opinion in Psychology, 16, 134-137. https://doi.org/10.1016/j.copsyc.2017.05.007

Torregrossa, M., Chamorro, J. L. y Ramis, Y. (2016). Transición de júnior a sénior y promoción de carreras duales en el deporte: una revisión interpretativa. Revista de Psicología Aplicada al Deporte y al Ejercicio Físico, 1(1), Artículo e6.

https://doi.org/10.5093/rpadef2016a6

Torregrossa, M., Viladrich, C., Ramis, Y., Azócar, F., Latinjak, A. T. y Cruz, J. (2011). Efectos en la percepción del clima motivacional generado por los entrenadores y compañeros sobre la 
diversión y el compromiso. Diferencias en función de género. Revista de Psicología del Deporte, 20(1), 243-255.

Weinberg, R. S. y Gould, D. (2019). Welcome to Sport and Exercise Psychology. En Autores (Eds.), Foundations of sport and exercise psychology (7th ed., pp. 3-14). Human Kinetics.

Wylleman, P. (2019). An organizational perspective on applied sport psychology in elite sport. Psychology of Sport and Exercise, 42, 89-99. https://doi.org/10.1016/j.psychsport.2019.01.008
Wylleman, P. y Lavallee, D. (2004). A developmental pespective on transitions faced by athletes. In M. Weiss (Ed.), Developmental sport and exercise psychology: A lifespan perspective (pp. 507527). Fitness Information Technology.

Wylleman, P. y Rosier, N. (2016). Holistic perspective on the development of elite athletes. En M. Raab, P. Wylleman, R. Seiler, A. Elbe y A. Hatzigeorgiadis (Eds.), Sport and exercise psychology research (pp. 269-288). Academic Press. 